\title{
COHN PATH ALGEBRAS HAVE INVARIANT BASIS NUMBER
}

\author{
GENE ABRAMS AND MÜGE KANUNI
}

\begin{abstract}
For any finite directed graph $E$ and any field $K$ we show that the Cohn path algebra $C_{K}(E)$ has the Invariant Basis Number property, moreover Invariant Matrix Number property.
\end{abstract}

For any directed graph $E$ and field $K$ the Leavitt path algebra $L_{K}(E)$ of $E$ with coefficients in $K$ has been the object of intense research focus since its introduction in 2005, see e.g. [1] and [6]. Leavitt path algebras are generalizations of the Leavitt algebras $L_{K}(1, n)(n \geq 2)$ introduced by Leavitt in [7]. A ring $R$ is said to have the Invariant Basis Number property (or more simply $I B N$ ) in case for any pair of positive integers $m \neq m^{\prime}$ we have that the free left $R$-modules $R^{m}$ and $R^{m^{\prime}}$ are not isomorphic. The Leavitt algebras fail to have the IBN property: for instance, if $R=L_{K}(1, n)$, then ${ }_{R} R^{1} \cong{ }_{R} R^{n}$. (Indeed, the search for nonIBN rings motivated much of Leavitt's work.) Additional examples abound of more general Leavitt path algebras which also fail to have the IBN property.

Let $M_{n}(R)$ denote $n \times n$ matrices over a ring $R$. We say that $R$ has the Invariant Matrix Number property, in case $M_{i}(R) \varsubsetneqq M_{j}(R)$ for every pair of positive integers $i \neq j$.

Recently, the Cohn path algebra $C_{K}(E)$ of a directed graph $E$ has been defined and investigated, see e.g., [2], 3], and/or [5]. As it turns out (see Theorem 5), for any directed graph $E$ there exists a directed graph $F$ for which $C_{K}(E) \cong L_{K}(F)$. Rephrased: every Cohn path algebra is in fact also a Leavitt path algebra. The purpose of this short note is to establish that, the abundance of non-IBN Leavitt path algebras notwithstanding, every Cohn path algebra has the Invariant Basis Number property, indeed, has the stronger Invariant Matrix Number property.

Throughout this note $E=\left(E^{0}, E^{1}, s, r\right)$ will denote a directed graph with vertex set $E^{0}$, edge set $E^{1}$, source function $s$, and range function $r$. In particular, the source vertex of

2010 Mathematics Subject Classification. Primary 16S99 Secondary 05C25.

Key words and phrases. Cohn path algebra, Leavitt path algebra, Invariant Basis Number.

The first author is partially supported by a Simons Foundation Collaboration Grants for Mathematicians Award \#208941. The second author is supported by a U.S. Department of State 2012-2013 Fulbright Visiting Scholar Program Grant and by the Scientific and Technological Research Council of Turkey (TÜBİTAKBIDEB) 2219 International Post-Doctoral Research Fellowship during her sabbatical visit to the University of Colorado Colorado Springs. This author would like to thank her colleagues at the host institution for their hospitality. 
an edge $e$ is denoted by $s(e)$, and the range vertex by $r(e)$. We will assume that $E$ is finite, i.e. that both $E^{0}$ and $E^{1}$ are finite sets. A sink is a vertex $v$ for which the set $s^{-1}(v)=\left\{e \in E^{1} \mid s(e)=v\right\}$ is empty; any non-sink is called a regular vertex.

For any directed graph $E$, we denote by $A_{E}$ the incidence matrix of $E$. Formally, if $E^{0}=\left\{v_{i} \mid 1 \leq i \leq n\right\}$, then $A_{E}=\left(a_{i, j}\right)$ is the $n \times n$ matrix for which $a_{i, j}$ is the number of edges $e$ having $s(e)=v_{i}$ and $r(e)=v_{j}$. In particular, if $v_{i} \in E^{0}$ is a sink, then $a_{i, j}=0$ for all $1 \leq j \leq n$, i.e., the $i^{\text {th }}$ row of $A_{E}$ consists of all zeros. We assume throughout that the vertices $\left\{v_{i} \mid 1 \leq i \leq n\right\}$ have been labelled in such a way that the vertices $\left\{v_{i} \mid 1 \leq i \leq t\right\}$ are the regular vertices, and $\left\{v_{i} \mid t+1 \leq i \leq n\right\}$ are the sinks (if any).

Definition 1. Let $K$ be a field, and let $E$ be a graph. The Cohn path $K$-algebra $C_{K}(E)$ of $E$ with coefficients in $K$ is the $K$-algebra generated by a set $\left\{v \mid v \in E^{0}\right\}$, together with a set of variables $\left\{e, e^{*} \mid e \in E^{1}\right\}$, which satisfy the following relations:

(V) $v w=\delta_{v, w} v$ for all $v, w \in E^{0}$

(E1) $s(e) e=e r(e)=e$ for all $e \in E^{1}$,

(E2) $r(e) e^{*}=e^{*} s(e)=e^{*}$ for all $e \in E^{1}$, and

$(\mathrm{CK} 1) e^{*} e^{\prime}=\delta_{e, e^{\prime}} r(e)$ for all $e, e^{\prime} \in E^{1}$.

The Leavitt path $K$-algebra $L_{K}(E)$ of $E$ with coefficients in $K$ is the $K$-algebra generated by the same set $\left\{v \mid v \in E^{0}\right\}$, together with the same set of variables $\left\{e, e^{*} \mid e \in E^{1}\right\}$, which satisfy the same set of relations (V), (E1), (E2), and (CK1), and also satisfy the additional relation

$(\mathrm{CK} 2) v=\sum_{\left\{e \in E^{1} \mid s(e)=v\right\}} e e^{*}$ for every regular vertex $v \in E^{0}$.

Specifically, if we let $N \subseteq C_{K}(E)$ denote the ideal of $C_{K}(E)$ generated by the elements of the form $v-\sum_{\left\{e \in E^{1} \mid s(e)=v\right\}} e e^{*}$, where $v \in E^{0}$ is a regular vertex, then we may view the Leavitt path algebra $L_{K}(E)$ as the quotient algebra $L_{K}(E) \cong C_{K}(E) / N$. For a finite graph $E$ we have that both $C_{K}(E)$ and $L_{K}(E)$ are unital, each having identity $1=\sum_{v \in E^{0}} v$. A perhaps-surprising additional connection between Cohn path algebras and Leavitt path algebras is given here; the discussion represents a specific case of a more general result described in [2, Section 1.5].

Definition 2. Let $K$ be a field, let $E$ be an arbitrary graph and let $Y$ denote the set of regular vertices of $E$. Let $Y^{\prime}=\left\{v^{\prime} \mid v \in Y\right\}$ be a disjoint copy of $Y$. For $v \in Y$ and for each edge $e$ in $E$ such that $r_{E}(e)=v$, we consider a new symbol $e^{\prime}$. We define the graph $F=F(E)$, as follows: $F^{0}=E^{0} \sqcup Y^{\prime} ; F^{1}=E^{1} \sqcup\left\{e^{\prime} \mid r_{E}(e) \in Y\right\}$; and for each $e \in E^{1}$, $s_{F}(e)=s_{E}(e), s_{F}\left(e^{\prime}\right)=s_{E}(e), r_{F}(e)=r_{E}(e)$, and $r_{F}\left(e^{\prime}\right)=r_{E}(e)^{\prime}$. 
Less formally, the graph $F=F(E)$ is built from $E$ by adding a new vertex to $E$ corresponding to each non-sink of $E$, and then including new edges to each of these new vertices in the same configuration as their counterpart vertices in $E$. Observe in particular that each of the new vertices $v^{\prime} \in Y^{\prime}$ is a sink in $F$. (We note that the graph $F(E)$ as defined here is the graph $E(\emptyset)$ of [2, Definition 1.5.15] when $E$ is finite.) The incidence matrix $A_{F}$ of $F=F(E)$ is the $(n+t) \times(n+t)$ matrix in which, for $1 \leq i \leq t$, the $i^{\text {th }}$ row is $\left(a_{i, 1}, a_{i, 2}, \ldots, a_{i, n}, a_{i, 1}, a_{i, 2}, \ldots, a_{i, t}\right)$ (where $A_{E}=\left(a_{i j}\right)$ is the incidence matrix of $E$ ), and the remaining $n$ rows are zeroes.

Example 3. Let $E$ be the graph $\bullet^{u} \stackrel{e}{\longrightarrow} \bullet^{v} \stackrel{f}{\longrightarrow} \bullet^{w}$. Then the graph $F=F(E)$ is:

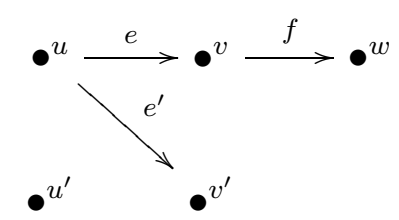

We note that there is no new vertex in $F$ corresponding to $w$, as $w$ is a sink in $E$.

Example 4. Let $R_{2}$ be the graph $\bigcup_{f}^{e}$. Then the graph $F=F\left(R_{2}\right)$ is:

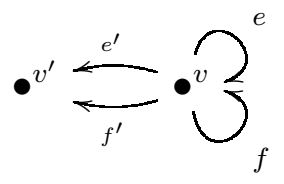

Here is the first key result we will utilize.

Theorem 5. (A specific case of [2, Theorem 1.5.17]) Let $E$ be any graph. Then there is an isomorphism of $K$-algebras $C_{K}(E) \cong L_{K}(F(E))$.

For any directed graph $E$ with $\left|E^{0}\right|=n$ we construct the abelian monoid $M_{E}$ as follows. Consider the abelian monoid $T=\left(\mathbb{Z}^{+}\right)^{n}$ of $n$-tuples of non-negative integers. For each regular vertex $v_{i}(1 \leq i \leq t)$ we let $\overrightarrow{b_{i}}$ denote the vector $(0,0, \ldots, 1,0, \ldots 0)$ (having 1 in the $i$-th component) of $\left(\mathbb{Z}^{+}\right)^{n}$. We consider the equivalence relation $\sim_{E}$ in $\left(\mathbb{Z}^{+}\right)^{n}$, generated by setting

$$
\overrightarrow{b_{i}} \sim_{E}\left(a_{i, 1}, a_{i, 2}, \ldots, a_{i, n}\right)
$$

for each regular vertex $v_{i}$. The monoid $M_{E}$ consists of the equivalence classes $\left(\mathbb{Z}^{+}\right)^{n} / \sim_{E}$; if we denote the equivalence class of an element $\vec{a} \in\left(\mathbb{Z}^{+}\right)^{n}$ by $[\vec{a}]$, then the operation in $M_{E}$ is given by setting $[\vec{a}]+\left[\overrightarrow{a^{\prime}}\right]=\left[\vec{a}+\overrightarrow{a^{\prime}}\right]$ for $\vec{a}, \overrightarrow{a^{\prime}} \in\left(\mathbb{Z}^{+}\right)^{n}$.

For instance, if $E$ is the graph of Example 3 , then $M_{E}$ is the monoid $\left(\mathbb{Z}^{+}\right)^{3}$, modulo the relation $\sim_{E}$ generated by setting $(1,0,0) \sim_{E}(0,1,0)$ and $(0,1,0) \sim_{E}(0,0,1)$. It is not hard 
to see that $M_{E} \cong \mathbb{Z}^{+}$. For the graph $F=F(E)$ of that same Example, one can show that $M_{F} \cong\left(\mathbb{Z}^{+}\right)^{3}$. On the other hand, if $R_{2}$ is the graph presented in Example 4, then $M_{R_{2}}$ is the monoid $\left(\mathbb{Z}^{+}\right)^{1}$, modulo the relation generated by setting $(1) \sim_{R_{2}}(2)$. In this case we see that $M_{R_{2}} \cong\{0, x\}$, where $x+x=x$. (N.b.: $M_{R_{2}}$ is not the group $\mathbb{Z}_{2}$.) Furthermore, for $F=F\left(R_{2}\right)$, one can show that $M_{F}$ is the monoid

$$
\{[(0, i)] \mid i \geq 0\} \sqcup\{[(1, i)] \mid i \geq 0\} \sqcup\{[(i, 0)] \mid i \geq 2\} \sqcup\{[(i, 1)] \mid i \geq 2\} .
$$

Observe in particular that for positive integers $m \neq m^{\prime},(m, 0) \nsim_{F}\left(m^{\prime}, 0\right)$ and $(m, 1) \nsim_{F}$ $\left(m^{\prime}, 1\right)$.

For any ring $R$, we denote by $\mathcal{V}(R)$ the abelian monoid of isomorphism classes of finitely generated projective left $R$-modules, with operation $\oplus$. If $P$ is a finitely generated projective left $R$-module, we denote the element of $\mathcal{V}(R)$ which contains $P$ by $[P]$. We recast the IBN property in $\mathcal{V}(R)$ as follows: $R$ has IBN if and only if for every pair of distinct positive integers $m \neq m^{\prime}$ we have $m[R] \neq m^{\prime}[R]$ as elements of $\mathcal{V}(R)$. Here is the second key result we will utilize.

Theorem 6. ([6, Theorem 3.5]) Let $E$ be a finite graph with vertices $\left\{v_{i} \mid 1 \leq i \leq n\right\}$, and let $K$ be any field. Then the assignment $\left[\overrightarrow{b_{i}}\right] \mapsto\left[L_{K}(E) v_{i}\right]$ yields an isomorphism of monoids $M_{E} \cong \mathcal{V}\left(L_{K}(E)\right)$. In particular, under this isomorphism, if $\vec{\rho}=(1,1, \ldots, 1) \in\left(\mathbb{Z}^{+}\right)^{n}$, we have $[\vec{\rho}] \mapsto\left[L_{K}(E)\right]$.

Consequently,

Corollary 7. Let $F$ be any finite graph, and $K$ any field. Let $\vec{\rho}=(1,1, \ldots, 1) \in M_{F}$. Then $L_{K}(F)$ has IBN if and only if for any pair of positive integers $m \neq m^{\prime}$, we have $m \vec{\rho} \nsim_{F} m^{\prime} \vec{\rho}$.

In the graph $E$ of Example 3, we have that $M_{E} \cong \mathbb{Z}^{+}$; moreover, in this identification, $[\vec{\rho}] \mapsto 3$. Since $m \neq m^{\prime}$ obviously gives $m \cdot 3 \neq m^{\prime} \cdot 3$ in $\mathbb{Z}^{+}$, we conclude that $L_{K}(E)$ has IBN. Further, in the graph $F(E)$ of that same Example, we have that $M_{F(E)} \cong\left(\mathbb{Z}^{+}\right)^{3}$, and in this identification we have $[\vec{\rho}] \mapsto(3,1,2)$. As before, $m \neq m^{\prime}$ obviously gives $m(3,1,2) \neq$ $m^{\prime}(3,1,2)$ in $\left(\mathbb{Z}^{+}\right)^{3}$, we conclude that $L_{K}(F(E))$ has IBN as well. On the other hand, if $R_{2}$ is the graph of Example 4 , then $M_{R_{2}} \cong\{0, x\}$, and in this identification we have $[\vec{\rho}] \mapsto x$. Since $1 x=2 x$, we conclude that $L_{K}\left(R_{2}\right)$ does not have IBN. (We note that each of these three observations is well-known, we have included them here only to help clarify Corollary [7. Indeed, $L_{K}\left(R_{2}\right) \cong L_{K}(1,2)$, the non-IBN Leavitt algebra for $n=2$ mentioned in the 
introductory paragraphs of the article.) Finally, for the graph $F=F\left(R_{2}\right)$, we get

$$
m \vec{\rho}=(m, m) \sim_{F} \begin{cases}\left(\frac{m}{2}, 0\right) & \text { if } m \text { is even } \\ \left(\frac{m+1}{2}, 1\right) & \text { if } m \text { is odd }\end{cases}
$$

for any positive integer $m$. So by a previous observation we get that $m \neq m^{\prime}$ gives $m \vec{\rho} \nsim_{F}$ $m^{\prime} \vec{\rho}$. Hence $L_{K}\left(F\left(R_{2}\right)\right)$ has IBN property. That both $L_{K}(F(E))$ and $L_{K}\left(F\left(R_{2}\right)\right)$ have the IBN property will also follow as a consequence of Theorem 9 .

We will use the following elementary linear algebra result.

Proposition 8. Given any non-negative integers $a_{i j}(1 \leq i \leq t, 1 \leq j \leq n)$, there exist $w_{1}, w_{2}, \ldots, w_{n+t}$ in $\mathbb{Q}$ which satisfy the following system of $t+1$ linear equations:

$$
\begin{aligned}
& 1=w_{1}+w_{2}+\ldots+w_{n}+w_{n+1}+\ldots+w_{n+t} \\
& w_{1}=a_{11} w_{1}+a_{12} w_{2}+\ldots+a_{1 n} w_{n}+a_{11} w_{n+1}+\ldots+a_{1 t} w_{n+t} \\
& w_{2}=a_{21} w_{1}+a_{22} w_{2}+\ldots+a_{2 n} w_{n}+a_{21} w_{n+1}+\ldots+a_{2 t} w_{n+t} \\
& \vdots \\
& w_{t}=a_{t 1} w_{1}+a_{t 2} w_{2}+\ldots+a_{t n} w_{n}+a_{t 1} w_{n+1}+\ldots+a_{t t} w_{n+t}
\end{aligned}
$$

Proof. Consider the following $(t+1) \times(n+t)$ matrix $B$ :

$$
B=\left(\begin{array}{ccccccccc}
1 & 1 & \ldots & 1 & \ldots & 1 & 1 & \ldots & 1 \\
a_{11}-1 & a_{12} & \ldots & a_{1 t} & \ldots & a_{1 n} & a_{11} & \ldots & a_{1 t} \\
a_{21} & a_{22}-1 & \ldots & a_{2 t} & \ldots & a_{2 n} & a_{21} & \ldots & a_{2 t} \\
\vdots & & & & & & & & \\
a_{t 1} & a_{t 2} & \ldots & a_{t t}-1 & \ldots & a_{t n} & a_{t 1} & \ldots & a_{t t}
\end{array}\right)
$$

Then the existence of rationals $w_{1}, w_{2}, \ldots, w_{n+t}$ which satisfy the indicated equations is equivalent to the existence of a solution in $\mathbb{Q}^{n+t}$ to the system of $t+1$ equations represented by the vector equation $B \vec{x}=(1,0,0, \ldots, 0)^{t}$.

We claim that the $t+1$ rows of $B$ are linearly independent. For $1 \leq i \leq t$ we subtract column $i$ from column $n+i$ in the matrix $B$, which yields the column-equivalent matrix $C$ :

$$
C=\left(\begin{array}{cccccccccc}
1 & 1 & \ldots & 1 & \ldots & 1 & 0 & 0 & \ldots & 0 \\
a_{11}-1 & a_{12} & \ldots & a_{1 t} & \ldots & a_{1 n} & 1 & 0 & \ldots & 0 \\
a_{21} & a_{22}-1 & \ldots & a_{2 t} & \ldots & a_{2 n} & 0 & 1 & \ldots & 0 \\
\vdots & & & & & & & & & \\
a_{t 1} & a_{t 2} & \ldots & a_{t t}-1 & \ldots & a_{t n} & 0 & 0 & \ldots & 1
\end{array}\right)
$$


The final $t+1$ columns of $C$ are clearly linearly independent, so that columnrank $(C) \geq t+1$. But columnrank $(C)=\operatorname{columnrank}(B)=\operatorname{rowrank}(B) \leq t+1$ (since $B$ has $t+1$ rows), so that $\operatorname{columnrank}(C)=\operatorname{rowrank}(B)=t+1$.

Since the $t+1$ rows of $B$ are linearly independent in $\mathbb{Q}^{n+t}$, there exists a solution to any system of equations of the form $B \vec{x}=\vec{c}$ for any $\vec{c}$ in $\mathbb{Q}^{t+1}$, in particular for $\vec{c}=(1,0,0, \ldots, 0)^{t}$.

We now have in place all the tools we need to establish our main result, that any Cohn path algebra $C_{K}(E)$ has IBN (Theorem 9). But prior to doing so, we make an observation which will provide some context. By Theorem [5, together with the specific construction of the graph $F=F(E)$, we see that $C_{K}(E)$ is isomorphic to a Leavitt path algebra $L_{K}(F)$ for which the graph $F$ necessarily has sinks. So one might be tempted to conjecture that our main result is simply an artifact of a result about Leavitt path algebras of graphs with sinks. But indeed there are numerous examples of graphs $G$ with sinks for which the Leavitt path algebra $L_{K}(G)$ does not have IBN; for instance the graph $G=E(X)$ described Example 12 below is such.

Here now is our main result.

Theorem 9. Let $E$ be any finite graph, and $K$ any field. Then the Cohn path algebra $C_{K}(E)$ has the Invariant Basis Number property.

Proof. By Theorem 5 we have $C_{K}(E) \cong L_{K}(F)$, where $F=F(E)$ is the graph described in Definition 2, Specifically, if $E^{0}=\left\{v_{1}, v_{2}, \ldots, v_{n}\right\}$, and we label the regular vertices of $E$ as $v_{1}, \ldots, v_{t}$, then $F^{0}=\left\{v_{1}, v_{2}, \ldots, v_{n}, v_{1}^{\prime}, v_{2}^{\prime}, \ldots, v_{t}^{\prime}\right\}$, and the only regular vertices of $F$ are $\left\{v_{1}, v_{2}, \ldots, v_{t}\right\}$. Note that $\left|F^{0}\right|=n+t$.

Let $A=A_{E}=\left(a_{i, j}\right)$ be the incidence matrix of $E$. Then the monoid $M_{F}$ is the monoid $\left(\mathbb{Z}^{+}\right)^{n+t}$, modulo the equivalence relation generated by setting

$$
\overrightarrow{b_{i}} \sim_{F}\left(a_{i, 1}, a_{i, 2}, \ldots, a_{i, n}, a_{i, 1}, a_{i, 2}, \ldots, a_{i, t}\right) \text { for each } 1 \leq i \leq t
$$

Let $\vec{\rho}$ denote the element $(1,1, \ldots, 1)$ of $\left(\mathbb{Z}^{+}\right)^{n+t}$. We establish, for any pair of positive integers $m \neq m^{\prime}$, that $m \vec{\rho} \nsim_{F} m^{\prime} \vec{\rho}$.

Let $w_{1}, w_{2}, \ldots, w_{n+t} \in \mathbb{Q}$ be rationals which satisfy the linear system presented in, and whose existence is guaranteed by, Proposition 8. In particular, for $1 \leq i \leq t$ we have $w_{i}=\sum_{\ell=1}^{n+t} a_{i, \ell} w_{\ell}$. We define the map $\Gamma:\left(\mathbb{Z}^{+}\right)^{n+t} \rightarrow \mathbb{Q}$ by setting

$$
\Gamma\left(\left(z_{1}, z_{2}, \ldots, z_{n+t}\right)\right)=\sum_{\ell=1}^{n+t} z_{\ell} w_{\ell} .
$$


Then $\Gamma$ is clearly linear. We claim that if $\left(z_{1}, z_{2}, \ldots, z_{n+t}\right) \sim_{F}\left(z_{1}^{\prime}, z_{2}^{\prime}, \ldots, z_{n+t}^{\prime}\right)$, then $\Gamma\left(\left(z_{1}, z_{2}, \ldots, z_{n+t}\right)\right)=\Gamma\left(\left(z_{1}^{\prime}, z_{2}^{\prime}, \ldots, z_{n+t}^{\prime}\right)\right)$. The equivalence relation $\sim_{F}$ is generated by the relations given in $(\dagger)$, so it suffices to show that the claim holds if we apply any one of these generating relations to $\left(z_{1}, z_{2}, \ldots, z_{n+t}\right)$ to produce $\left(z_{1}^{\prime}, z_{2}^{\prime}, \ldots, z_{n+t}^{\prime}\right)$. That is, it suffices to establish the claim in the situation where $\left(z_{1}, z_{2}, \ldots, z_{n+t}\right)=\vec{a}+\overrightarrow{b_{i}}$ and $\left(z_{1}^{\prime}, z_{2}^{\prime}, \ldots, z_{n+t}^{\prime}\right)=$ $\vec{a}+\left(a_{i, 1}, a_{i, 2}, \ldots a_{i, n}, a_{i, 1}, a_{i, 2}, \ldots a_{i, t}\right)$, for each $1 \leq i \leq t$, and for any $\vec{a} \in\left(\mathbb{Z}^{+}\right)^{n+t}$. Using the definition and linearity of $\Gamma$, we get

$$
\begin{aligned}
\Gamma\left(\left(z_{1}, z_{2}, \ldots, z_{n+t}\right)\right) & =\Gamma\left(\vec{a}+\overrightarrow{b_{i}}\right)=\Gamma(\vec{a})+\Gamma\left(\overrightarrow{b_{i}}\right)=\Gamma(\vec{a})+1 w_{i}=\Gamma(\vec{a})+\sum_{\ell=1}^{n+t} a_{i, \ell} w_{\ell} \\
& =\Gamma(\vec{a})+\Gamma\left(\left(a_{i, 1}, a_{i, 2}, \ldots a_{i, n}, a_{i, 1}, a_{i, 2}, \ldots a_{i, t}\right)\right) \\
& =\Gamma\left(\left(z_{1}^{\prime}, z_{2}^{\prime}, \ldots, z_{n+t}^{\prime}\right)\right) .
\end{aligned}
$$

Thus we have established the claim.

But recall that, by Proposition 8 , the $w_{\ell}$ have been chosen so that $\sum_{\ell=1}^{n+t} w_{\ell}=1$. So in particular for any positive integer $m$ we get

$$
\Gamma(m \vec{\rho})=\Gamma((m, m, \ldots, m))=\sum_{\ell=1}^{n+t} m w_{\ell}=m \sum_{\ell=1}^{n+t} w_{\ell}=m \cdot 1=m .
$$

So for $m \neq m^{\prime}$ we have $\Gamma(m \vec{\rho})=m \neq m^{\prime}=\Gamma\left(m^{\prime} \vec{\rho}\right)$, so that by the contrapositive of the claim we conclude that if $m \neq m^{\prime}$, then $m \vec{\rho} \nsim_{F} m^{\prime} \vec{\rho}$. Now Corollary 7 completes the proof.

As one consequence of our main result, we state that any Cohn path algebra over a finite graph will also have Invariant Matrix Number. This result follows from the next proposition that we recall from [4].

Proposition 10. ([4, Proposition 4]) If $F$ is a finite graph, and $\left[L_{K}(F)\right]$ has infinite order in $K_{0}\left(L_{K}(F)\right)$, then $L_{K}(F)$ has Invariant Matrix Number.

Corollary 11. Let $E$ be any finite graph, and $K$ any field. Then the Cohn path algebra $C_{K}(E)$ has Invariant Matrix Number.

Proof. Using the same terminology and setup as in the proof of the main theorem, we will show that $C_{K}(E) \cong L_{K}(F)$ has Invariant Matrix Number.

Let $R$ be any unital ring, and suppose that $R$ has finite order in $K_{0}(R)$. Then $n[R]=[0]$ in $K_{0}(R)$ for some $n \in \mathbb{N}$. By the construction of $K_{0}$, this means that $R^{n}$ is stably isomorphic to $\{0\}$; i.e., that there exists $m \in \mathbb{N}$ with $R^{n} \oplus R^{m} \cong\{0\} \oplus R^{m}$ as left $R$-modules. But then 
$R^{n+m} \cong R^{m}$ as left $R$-modules. The upshot of this discussion is that for any unital ring $R$ having Invariant Basis Number, then $[R]$ has infinite order in $K_{0}(R)$.

Thus Theorem 2.2 together with Proposition 2.2 apply to yield the result.

The Cohn path algebra $C_{K}(E)$ and the Leavitt path algebra $L_{K}(E)$ of a graph $E$ may be thought of as occupying two ends of a spectrum: to build $L_{K}(E)$ we impose the (CK2) relation at all of the vertices of $E$, whereas to build $C_{K}(E)$ we impose the (CK2) relation at none of the vertices. There is intermediate ground: for any set $X \subseteq \operatorname{Reg}(E)$ we define the relative Cohn path algebra $C_{K}^{X}(E)$ to be the $K$-algebra generated by the same relations as the relations which generate $C_{K}(E)$, where in addition we impose the (CK2) relation precisely at the vertices in $X$. (See [2, Section 5] for additional information.) So $C_{K}(E)=C_{K}^{\emptyset}(E)$, while $L_{K}(E)=C_{K}^{\operatorname{Reg}(E)}(E)$. Cast in the context of Theorem 9, the case $X=\emptyset$ (i.e, $|X|=0$ ) plays a unique role, in this sense: for any positive integers $1 \leq m \leq n$ there exists a graph $E$ and $X \subseteq E^{0}$ having $\left|E^{0}\right|=n,|X|=m$, and for which $C_{K}^{X}(E)$ does not have IBN. We describe here such a graph in the case $m=1, n=2$; the construction easily generalizes to arbitrary $m, n$.

Example 12. Let $E$ be the graph $\bigodot_{\bullet} \longleftarrow \bullet \overbrace{}^{2}$, and let $X=\{w\}$. By [2, Theorem 1.5.17], $C_{K}^{X}(E) \cong L_{K}(E(X))$, where the graph $E(X)$ is $\left(\mathbb{Z}^{+}\right)^{3}$ modulo the equivalence relation $\sim_{M(E)}$ generated by setting $(1,0,0) \sim_{E(X)}(1,0,1)$ and $(0,1,0) \sim_{E(X)}(1,2,1)$. But then $\vec{\rho}=(1,1,1)=(1,0,1)+(0,1,0) \sim_{E(X)}(1,0,1)+(1,2,1)=$ $(2,2,2)=2 \vec{\rho}$, so that $L_{K}(E(X))$, and thus also $C_{K}^{X}(E)$, does not have the IBN property by Corollary 7. For the general case, Let $E_{n}$ be the graph

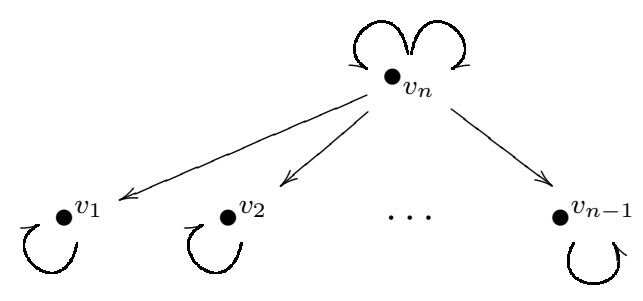

and $X_{m}=\left\{v_{n-m+1}, \ldots, v_{n}\right\}$. Then using the same ideas as for the graph $E$ above, it is easy to establish that $C_{K}^{X_{m}}\left(E_{n}\right)$ does not satisfy the IBN property.

We want to point out that, in $L_{K}(F)$ with IBN property, for $m \neq m^{\prime}$, although $m \vec{\rho} \chi_{F} m^{\prime} \vec{\rho}$, there can exist elements $x$ in $M_{F}$ for which $m x \sim_{F} m^{\prime} x$. Take for instance, the graph $F=$ 
$F\left(R_{2}\right)$ of Example 4 and consider the element $x=(1,2)$. Then $x=(1,2) \sim_{F}(2,2)+(0,2)=$ $(2,4)=2 x$.

We conclude this short note by providing an alternate proof of the main result. Unlike the approach used above (an 'invariant-based' method), this alternate approach makes use of an explicit description of $\mathcal{V}\left(C_{K}(E)\right)$, together with a result about monoids generated by relations of a specified type. This alternate approach was suggested by P. Ara, to whom the authors are extremely grateful.

Theorem 9, re-established. We define the monoid $M_{C(E)}$ as follows. Let $T$ denote the free abelian monoid (written additively) with generators $E^{0} \sqcup\left\{q_{v} \mid v\right.$ is regular $\}$. Define relations in $T$ by setting

$$
v=q_{v}+\sum_{e \in s^{-1}(v)} r(e)
$$

for each $v \in \operatorname{Reg}(E)$. Let $\sim$ be the equivalence relation in $T$ generated by these relations. Then $M_{C(E)}=T / \sim$, with operation defined by by setting $[x]+[y]=[x+y]$.

By [5, Theorem 4.3], $\mathcal{V}\left(C_{K}(E)\right) \cong M_{C(E)}$; moreover, under this isomorphism, $\left[C_{K}(E)\right] \mapsto$ $\left[\sum_{v \in E^{0}} v\right]$. We denote $E^{0}$ by $\left\{v_{1}, v_{2}, \ldots, v_{n}\right\}$. Thus we achieve the desired conclusion by showing that, for positive integers $m, m^{\prime}$, if $m\left[\sum_{i=1}^{n} v_{i}\right]=m^{\prime}\left[\sum_{i=1}^{n} v_{i}\right]$ in $M_{C(E)}$, then $m=m^{\prime}$.

For each $t \in T$ Let $\ddagger_{i}(t)$ denote the element of $T$ which results by applying the relation $(\ddagger)$ corresponding to vertex $v_{i}$ on $t$. For any sequence $\sigma$ (of any length) taken from $\{1,2, \ldots, n\}$, and any $t \in T$, let $\Lambda_{\sigma}(t) \in T$ be the element which results by applying $\ddagger_{i}$ operations in the order specified by $\sigma$.

The monoid $M_{C(E)}$ is given by generators and relations of the form described in [6, Section 4] (where the monoid is denoted by $F$ ). In particular, by [6, Lemma 4.3] (the so-called 'Confluence Lemma'), the hypothesis $m\left[\sum_{i=1}^{n} v_{i}\right]=m^{\prime}\left[\sum_{i=1}^{n} v_{i}\right]$ in $M_{C(E)}$ yields that there are two sequences $\sigma, \sigma^{\prime}$ for which

$$
\Lambda_{\sigma}\left(m \sum_{i=1}^{n} v_{i}\right)=\gamma=\Lambda_{\sigma^{\prime}}\left(m^{\prime} \sum_{i=1}^{n} v_{i}\right)
$$

for some $\gamma \in T$. But each time a substitution of the form $\ddagger_{i}$ is made to an element of $T$, the effect on that element is to:

(i) subtract 1 from the coefficient on $v_{i}$;

(ii) add $a_{i j}$ to the coefficient on $v_{j}$ (for $1 \leq j \leq n$ ); and

(iii) add 1 to the coefficient on $q_{v_{i}}$.

As one consequence of this observation we see that, starting with $t \in T$ for which the coefficient on each $q_{v_{i}}$ in $t$ is 0 , the coefficient on $q_{v_{i}}$ in $\Lambda_{\sigma}(t)$ is precisely the number of times 
the relation $\ddagger_{i}$ was invoked. In particular, since both $m \sum_{i=1}^{n} v_{i}$ and $m^{\prime} \sum_{i=1}^{n} v_{i}$ have this property, the equation $\Lambda_{\sigma}\left(m \sum_{i=1}^{n} v_{i}\right)=\Lambda_{\sigma^{\prime}}\left(m^{\prime} \sum_{i=1}^{n} v_{i}\right)$ in $T$ yields that for each regular vertex $v_{i}$, the number of times $\ddagger_{i}$ is invoked in $\Lambda_{\sigma}$ is the same as the number of times that $\ddagger_{i}$ is invoked in $\Lambda_{\sigma^{\prime}}$. Denote this common number by $k_{i}$. Recalling the previously observed effect of $\ddagger_{i}$ on any element of $T$, we see that

$$
\begin{aligned}
\gamma= & \Lambda_{\sigma}\left(m \sum_{i=1}^{n} v_{i}\right) \\
= & \left(\left(m-k_{1}\right)+k_{1} a_{11}+k_{2} a_{21}+\ldots+k_{n} a_{n 1}\right) v_{1} \\
& +\left(\left(m-k_{2}\right)+k_{1} a_{12}+k_{2} a_{22}+\ldots+k_{n} a_{n 2}\right) v_{2}+\ldots \\
& +\left(\left(m-k_{n}\right)+k_{1} a_{1 n}+k_{2} a_{2 n}+\ldots+k_{n} a_{n n}\right) v_{n}+k_{1} q_{v_{1}}+k_{2} q_{v_{2}}+\cdots+k_{n} q_{v_{n}} .
\end{aligned}
$$

But then also

$$
\begin{aligned}
\gamma= & \Lambda_{\sigma^{\prime}}\left(m^{\prime} \sum_{i=1}^{n} v_{i}\right) \\
= & \left(\left(m^{\prime}-k_{1}\right)+k_{1} a_{11}+k_{2} a_{21}+\ldots+k_{n} a_{n 1}\right) v_{1} \\
& +\left(\left(m^{\prime}-k_{2}\right)+k_{1} a_{12}+k_{2} a_{22}+\ldots+k_{n} a_{n 2}\right) v_{2}+\ldots \\
& +\left(\left(m^{\prime}-k_{n}\right)+k_{1} a_{1 n}+k_{2} a_{2 n}+\ldots+k_{n} a_{n n}\right) v_{n}+k_{1} q_{v_{1}}+k_{2} q_{v_{2}}+\cdots+k_{n} q_{v_{n}}
\end{aligned}
$$

By equating coefficients on any of the free generators $v_{i}$ of $T$, we conclude that $m-k_{i}=$ $m^{\prime}-k_{i}$, so that $m=m^{\prime}$ as desired.

\section{REFERENCES}

[1] G. Abrams and G. Aranda Pino, The Leavitt path algebra of a graph, J. Algebra 293(2) (2005), 319-334.

[2] G. Abrams, P. Ara, and M. Siles Molina Leavitt path algebras. Lecture Notes in Mathematics series, Springer-Verlag Inc. (to appear).

[3] G. Abrams and Z. Mesyan, Simple Lie algebras arising from Leavitt path algebras, J. Pure Applied Algebra 216(10) (2012), 2302-2313.

[4] G. Abrams and C. Smith, Matrix type of purely infinite simple Leavitt path algebras, Acta Mathematica Hungarica 128(4) (2010), 381-385.

[5] P. Ara and K. Goodearl, Leavitt path algebras of separated graphs, J. Reine Angew. Math. 669 (2012), $165-224$.

[6] P. Ara, M.A. Moreno, And E. Pardo, Nonstable K-theory for graph algebras, Alg. Rep. Thy. 10 (2007), 157-178.

[7] W. Leavitt, The module type of a ring. Trans. Amer. Math. Soc. 103 (1962), 113-130. 
Department of Mathematics, University of Colorado, Colorado Springs CO 80918 U.S.A. E-mail address: abrams@math.uccs.edu

Department of Mathematics, Düzce University, Konuralp Düzce 81620 Turkey

E-mail address: mugekanuni@duzce.edu.tr 